\title{
TRIBUNA DE OPINIÓN: EMPRENDIMIENTO Y UNIVERSIDAD. UNA REFERENCIA AL CASO DE ESPAÑA Y A LA UPV.
}

POR

Juan Francisco JULIÀ ${ }^{1}$

\section{RESUMEN}

El emprendimiento entendido de forma amplia como el desarrollo de una idea o negocio, principalmente en el ámbito empresarial, es hoy sin duda un fenómeno de gran interés y especialmente si éste es innovador.

Los países más competitivos y las economías más desarrolladas se distinguen por contar con un tejido productivo que tiene su origen en un emprendimiento que podemos llamar inteligente, ya que está basado en uso innovador del conocimiento y la creatividad, y en tecnologías avanzadas, que muestra además gran capacidad de mercado y de internacionalización.

En este sentido, se observa la importancia de la búsqueda de ecosistemas en los que se reúnan el conocimiento, la creatividad y la tecnología, que permitan mejorar la capacidad innovadora desde el emprendimiento.

Las universidades, y muy especialmente las de perfiles científico y tecnológico pueden constituir a tal fin un ecosistema muy favorable y contribuir al necesario cambio hacia una economía como la de los países más avanzados y competitivos, basada en el conocimiento. Esté, es sin duda, hoy el reto de una economía como la española, y ésta es la visión que se tiene desde una universidad tecnológica española de cómo la Universitat Politècnica de València, líder en innovación y transferencia, de cómo se puede contribuir desde una

\footnotetext{
${ }^{1}$ Juan Francisco Juliá: Presidente de la Fundación Ciudad Politécnica de la Innovación CEGEA. Universitat Politècnica de València (jfjulia@esp.upv.es)

REVESCO No 113 - MONOGRÁFICO: Crisis económica actual y sus posibles repercusiones en la economía social - ISSN: 1885-8031 - www.ucm.es/info/revesco 
institución académica universitaria a generar un ecosistema de emprendimiento inteligente y con valores.

Palabras clave: competitividad, economía del conocimiento, economía social, economía con valores, emprendimiento, innovación, universidad.

\title{
TRIBUNE OPINION : ENTREPRENEURSHIP AND UNIVERSITY. A REFERENCE TO THE CASE OF SPAIN AND THE UPV.
}

\begin{abstract}
The project broadly understood as the development of an idea or business, mainly in business, is now definitely a phenomenon of great interest, especially if it is innovative.

The most competitive countries and most developed economies are distinguished by having a productive network that has its origin in a venture we call smart because it is based on innovative use of knowledge and creativity, and advanced technologies which also shows great ability to market and internationalization.
\end{abstract}

In this way, we observe the importance of finding the ecosystems where knowledge, creativity and technology to improve the innovative capacity from the enterprise.

Universities, and especially the scientific and technological profiles for this purpose can be a very favorable ecosystem and contribute to the necessary shift towards an economy like that of the most advanced and competitive countries, based on knowledge. It is certainly today the challenge of an economy like the Spanish, and this is the view we have from a Spanish University of Technology and how the Technical University of Valencia, a leader in innovation and transfer, to contribute from a university to create an ecosystem of intelligent Enterprise and values academic institution.

Keywords: competitiveness, knowledge economy, social economy, economy values, entrepreneurship, innovation, university.

\section{CONCEPTO Y VISIÓN ACTUAL DEL EMPRENDIMIENTO}

Emprendimiento es un término que se escucha y lee habitualmente en los medios en estos últimos años y que sin duda suscita mucho interés, con una valoración en general muy

REVESCO $\mathrm{N}^{\circ} 113$ - MONOGRÁFICO: Crisis económica actual y sus posibles repercusiones en la economía social - ISSN: 1885-8031 - www.ucm.es/info/revesco 
positiva. Sin embargo, es un término conceptualmente manejado de forma muy amplia y no muy precisa ya que admite varios usos más o menos limitados.

La palabra deriva de la voz castellana "emprender" que proviene del latín colligere o tomar, siendo también generalmente aceptado que cuando se habla de emprender este verbo deriva del vocablo "entrepreneur", que se usa para describir a un individuo que organiza y opera en una empresa asumiendo riesgo financiero para hacerlo, ligándolo claramente a la figura del emprendedor empresarial.

No obstante, es claro que el concepto puede y es de hecho utilizado en ocasiones en un sentido mucho más amplio, entendiéndolo como el hecho de implementar o desarrollar una idea o negocio. Así la Real Academia de la Lengua Española incorporará en su próxima edición este término, con el significado de acción y efecto de emprender (acometer una obra) y cualidad de emprendedor.

Y se señala asimismo que el uso del término emprendedurismo es una traducción inadecuada de la palabra entrepreneurship usada para describir el espíritu emprendedor en sentido empresarial.

Lo cierto es que el término emprendimiento se le acompaña muchas veces de adjetivos que dan muestra de su uso generalizado. Así, se habla de emprendimiento social, emprendimiento femenino, emprendimiento verde, emprendimiento por cuenta ajena o intraemprendimiento, emprendimiento propio, etc.

En todo caso, sí participamos, de la idea que el hecho de emprender siempre debería relacionarse con la innovación en el sentido en el que señalaban grandes economistas como Joseph Schumpeter o Peter Drucker que vinculan a los entrepreneurs a la innovación y los procesos de cambio (Schumpeter 1968, Drucker 1997).

Es cierto que la visión hoy del emprendimiento, como señalaba recientemente en una conferencia el Presidente de Redemprendia, profesor Senén Barro, como oportunidad y necesidad es ampliamente compartida, y que su uso como decíamos más común es el relacionado con la puesta en marcha de un negocio o empresa, que en nuestra opinión debería contar con un componente innovador, ya que como éste apunta, es una función previa a la innovación, recordando como señalaba Schumpeter que los progresos económicos son el resultado de innovaciones (Barro 2013).

REVESCO No 113 - MONOGRÁFICO: Crisis económica actual y sus posibles repercusiones en la economía social - ISSN: 1885-8031 - www.ucm.es/info/revesco 
¿Pero, cual es hoy la realidad hoy del emprendimiento en España?

En una primera aproximación y según el informe conocido como GEM 2012 que corresponde a la siglas de Global Entrepreneurship Monitor (GEM 2012), se cifra en 1.751.000 individuos representando este número, si hablamos de lo que se conoce por tasa de actividad emprendedora, esto es iniciativas activas (negocios) que van desde los 0 a 3 meses y de los 3 meses a los 42 meses, el 5,8 \% de la población entre 18 y 64 años, con un descenso de un $2 \%$ en relación con el año anterior, que hasta la crisis presentaba una tendencia en general de crecimiento positiva, pero que desde ya el 2008 ha sido a la inversa. Cabe señalar que si hablamos de emprendimiento potencial, esto es de aquellos individuos que durante los próximos tres años llevan idea de iniciar un negocio o actividad, y que arroja una tasa del $12 \%$, presenta al menos frente al pasado año un crecimiento de un $24 \%$ que resulta en principio esperanzador, aunque lo importante no es la cifra estadística sino los resultados económicos y sociales.

De hecho, lo que distingue a los países más competitivos no son estas cifras sino las características de su emprendimiento que serán determinantes en sus logros y su contribución a la competitividad de sus economías.

El perfil del emprendedor es desde luego variado, si bien ha crecido el de aquellos que cuentan con estudios superiores $34,4 \%$ y posgrados $6,6 \%$, situándose en más de un $40 \%$ los que cuentan con estudios universitarios o asimilables, y con un crecimiento frente al año anterior de un $13,6 \%$, que podemos valorar en positivo.

Eso sí, continua tratándose en general de microempresas (menos de 10 empleados) ya que un 93, 5\% contratan de 5 a ningún empleo ajeno (de 5 a 1 el 36,4\% y ninguno el 57,1\%) cifras que si bien mejoran frente al año anterior son ciertamente bajas, como el nivel medio de la inversión inicial que se sitúa en $18000 €$ y que con las dificultades de acceso a la financiación con motivo de la crisis que ha reducido la oferta crediticia, pese a la aparición y crecimiento de las capital riesgo, business angels, y demás, ha disminuido frente a años anteriores (Figura 1).

Del mismo modo, el porcentaje de emprendimiento que viene derivado como principal motivación por la necesidad, frente a la oportunidad de negocio, es todavía bastante relevante

REVESCO No 113 - MONOGRÁFICO: Crisis económica actual y sus posibles repercusiones en la economía social - ISSN: 1885-8031 - www.ucm.es/info/revesco 
ya que se sitúa sobre el $25,6 \%$, uno de cada cuatro, lo que es un valor superior al de los países más competitivos.

Con todo, la peor cara de nuestro emprendimiento viene de su carácter poco innovador, tan solo es un $19,2 \%$ y algo innovador un $22,6 \%$, eso sí con un crecimiento en torno al 20\% respecto al año anterior (Figura 1).

Otro tanto puede señalarse de su nivel tecnológico que es escaso, ya que atendiendo a la antigüedad de la principal tecnología utilizada nos encontramos que emplean tecnología de última generación de menos de 1 año solo el 12,5\% y de entre 1 y 5 años un 19,1 \% (Figura 1).

\section{Figura 1}

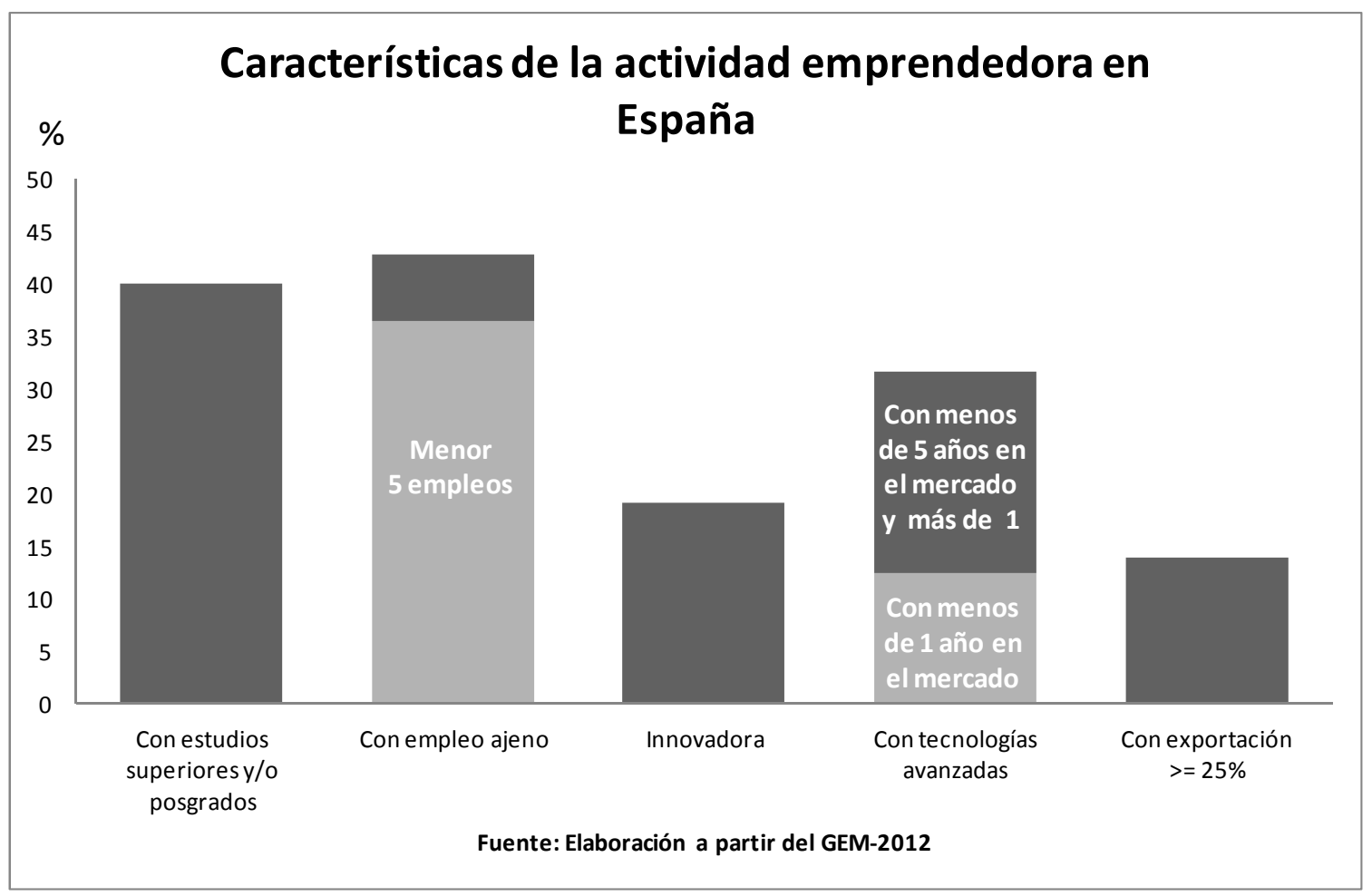

Ambos valores deben sin duda preocuparnos si atendemos a la estrecha relación que hay entre economías competitivas, e innovadoras y tecnológicamente avanzadas, y más cuando en la nuestra, la economía española, presenta aun hoy indicadores no precisamente positivos, así como nos recordaba el informe COTEC de este año (COTEC 2013), nuestro índice sintético de innovación (0,44 en el 2011 frente a por ejemplo Suiza 0,83 según la Comisión Europea2012) nos sitúa en el grupo de países innovadores moderados, lejos de los

REVESCO No 113 - MONOGRÁFICO: Crisis económica actual y sus posibles repercusiones en la economía social - ISSN: 1885-8031 - www.ucm.es/info/revesco 
líderes de innovación. Lo mismo que nuestro nivel tecnológico, aun bajo, y que lleva a que nuestra balanza comercial de bienes de alta y media tecnología sea muy deficitaria, con un nivel de empleo en alta y media cualificación inferior el de los países más desarrollados (Figura 2).

\section{Figura 2}

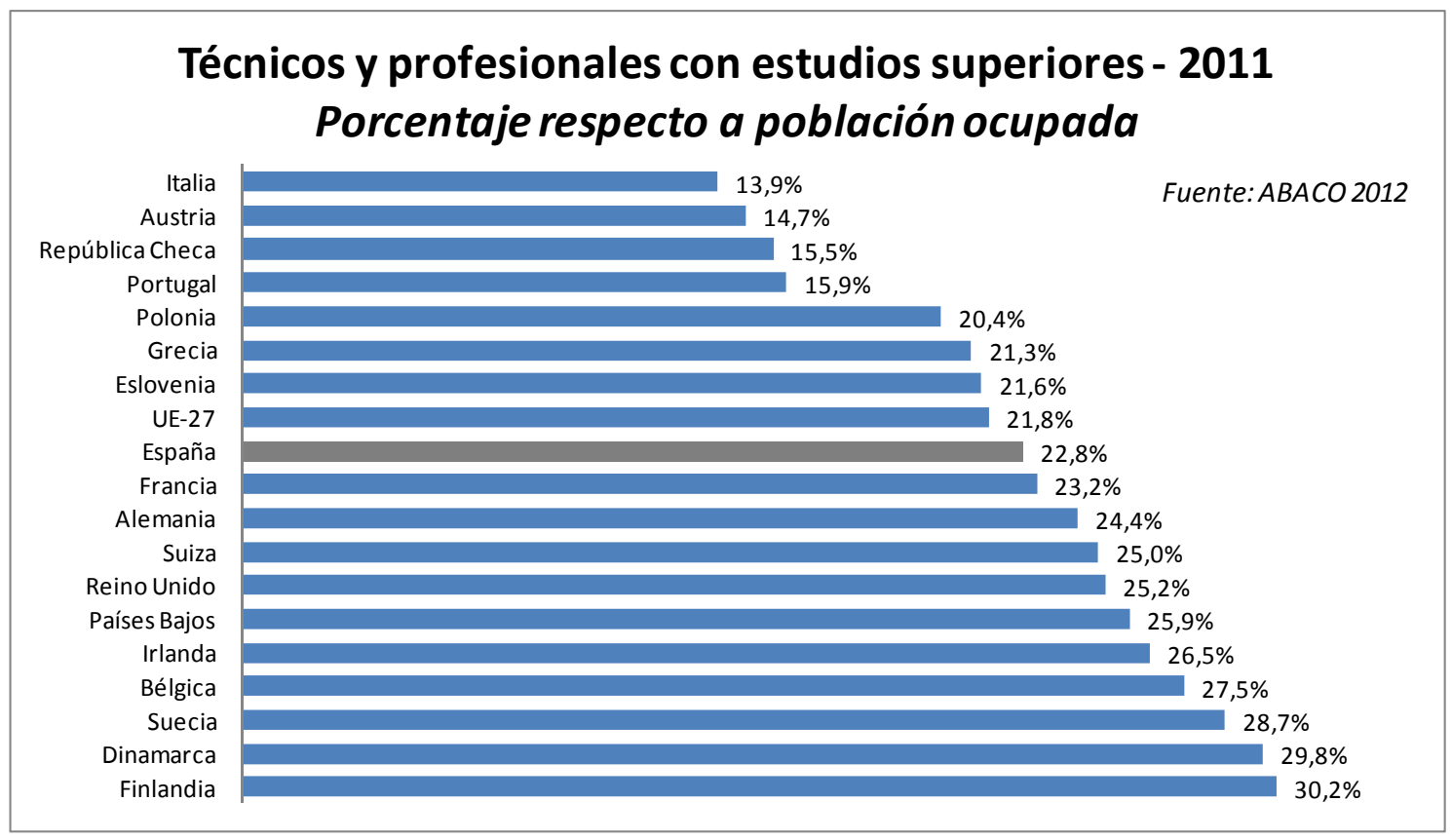

Tampoco en una economía global y abierta como la actual puede satisfacernos el contar con un emprendimiento en general poco internacionalizado, ya que nada menos que el $74,5 \%$ no exporta nada y tan solo exportan más de un 25\% de su producción, un 14\% (GEM 2012).

Sí es verdad, como señalaba el Profesor Federico Gutiérrez-Solana quien fue Presidente de la CRUE y actualmente dirige el Centro Internacional Santander de Emprendimiento (Gutiérrez-Solana 2013), que poseemos conocimiento, ya que nuestra producción científica es buena, se sitúa entre las primeras del mundo y es razonable en cuanto a calidad, produciéndose la mayor parte de ella en el sistema universitario público, con un nivel de inversión y gasto en I+D muy inferior al que correspondería a nuestra economía en términos de PIB, especialmente si nos referimos a la iniciativa privada.

También nuestra capacidad creativa es aceptable si atendemos al Global Creativity Index, que refleja que somos más creativos que competitivos.

REVESCO No 113 - MONOGRÁFICO: Crisis económica actual y sus posibles repercusiones en la economía social - ISSN: 1885-8031 - www.ucm.es/info/revesco 
Si es cierto como dice, que nuestro nivel de transferencia es bajo si atendemos a nuestro nivel de patentes y su explotación, que aún es menor si nos referimos al que correspondería a las empresas, y que explica nuestra baja capacidad y actividad innovadora, que por cierto según el último informe COTEC disminuyo el número de empresas con actividad innovadora (COTEC 2013), hechos que determinan nuestro bajo índice de competitividad que nos sitúa según el The Global Competitiveness Report en el puesto 36.

Ello nos hace pensar, que al menos si en el entorno del ecosistema universitario público, y dado el peso ya del emprendimiento de universitarios, se impulsa éste junto a la transferencia e innovación, cabe esperar una mejora de estos indicadores y con ellos de nuestra competitividad.

\section{INTERÉS Y OPORTUNIDAD DEL EMPRENDIMIENTO PARA LA ECONOMÍA ESPAÑOLA}

Una diferencia entre los sistemas de emprendimiento más exitosos y los que no lo son tanto, deriva de la visión de interés y oportunidad que tienen a la hora de emprender y del menor peso que tiene la mera necesidad como la motivación principal, siendo así por lo que preferimos abordar el tema desde este doble enunciado.

Comenzaremos para mostrar el interés del emprendimiento como valor social y económico, recordando que la primera potencia mundial y una de las cinco más competitivas e innovadoras es EEUU, donde su economía y sociedad conforman uno de los ecosistemas más favorables al emprendimiento. Alan Salomont, anterior Embajador de EEUU en España, y quiero recordar que fue quien llevó adelante la creación del primer American Space en España ubicándolo en Valencia en la Universidad Politécnica, decía que en su país se entiende que lo que es bueno para los emprendedores es bueno para el País, por ello se valora y se apoya como en ningún otro sitio con un marco legal y financiero muy favorable a todos quienes emprenden. Frases suyas como “ en ningún otro sitio del mundo han favorecido más a los emprendedores" y " América ama a sus emprendedores" testimonian bien lo dicho y explican, por ejemplo, que una de las revistas de más difusión: Times Magazine, tomara como portada de personajes del año a emprendedores como, Ted Turner (CNN), Andy Grove (Intel), Jeffrey Preston (Amazon), y más recientemente Bill Gates (Microsoft) y Mark Zuckerberg (FacebooK), en quien reciéntenme se basó una de las películas más taquilleras de

REVESCO No 113 - MONOGRÁFICO: Crisis económica actual y sus posibles repercusiones en la economía social - ISSN: 1885-8031 - www.ucm.es/info/revesco 
los últimos años, “La Red Social” y que muestra que el interés por el emprendimiento llega hasta la meca del cine, Hollywood (Salomont 2013).

Pero aquí, lo que nos interesa resaltar es que esto ha sido posible sin duda por su capacidad previa de innovación en el marco de una verdadera economía del conocimiento. Pelo Salaburu nos recuerda como la revista The economist señalaba que la cantidad y calidad de la vida intelectual en América (en referencia a EEUU) es también la más alta del mundo. Este País produce 1/3 de los artículos científicos, es donde trabajan 2/3 de los Premios Nobel, sitúa a 17 de sus universidades entre las 20 mejores universidades del mundo según el conocido ranking de Shanghai (Salaburu 2006).

Y es que también parafraseando a Salamont, "América ama a sus universidades", basta recordar la pasada campaña electoral a la Presidencia, cuando el actual Presidente Barak Obama decía en su discurso que sus universidades son una de la tres cosas de las que el pueblo americano se puede sentir más orgulloso".

Es bien claro el interés que supone el emprendimiento unido a innovación y conocimiento, es algo que sin duda contribuye e identifica a un país verdaderamente competitivo y con una fuerte economía.

Por otro lado, inevitablemente hemos de recordar que con toda seguridad el final de la primera década del siglo XXI y el inicio de la segunda, serán recordados en nuestra historia por la grave crisis económica global sin parangón en décadas pasadas. Una situación que se inicia con la crisis de las hipotecas basura, las conocidas como subprime, que puso de manifiesto los excesos del sector inmobiliario y financiero con una afición voraz a la especulación y donde hubo importantes fallos de los mecanismos de ordenación y regulación de los mercados, y una cuestión mucho más de fondo, una profunda crisis de valores dando prevalencia al imperio de la codicia y la sinrazón frente al interés social y económico.

Es verdad que en su desarrollo, esta crisis ha tenido y tiene ritmos e intensidades bien distintas. Así reviste una especial gravedad en algunas de las economías periféricas en Europa, especialmente en las del Sur, como en nuestro caso y ha servido para poner de manifiesto la necesidad de una economía más plural y equilibrada y sobre todo más competitiva.

REVESCO No 113 - MONOGRÁFICO: Crisis económica actual y sus posibles repercusiones en la economía social - ISSN: 1885-8031 - www.ucm.es/info/revesco 
La opción hasta la fecha por un modelo empresarial dominante y especulativo centrado casi exclusivamente en unos sectores (ladrillo, turismo) que en general no destacan por ser intensivos en conocimiento, ha determinado en buena medida el abandono de la necesaria atención hacia otros y ha llevado no solo al estallido de la burbuja inmobiliaria y la crisis financiera sino también a nuestra pérdida de productividad y por ende competitividad, cuyo camino de recuperación no puede basarse tan solo en abaratar costes (sobre todo de la mano de obra), sino en su mejora por el uso activo e inteligente del conocimiento, creatividad y tecnología, con un emprendimiento innovador. Esa y no otra es nuestra oportunidad.

Nuestra economía necesita de cambios ciertamente importantes. Debemos atajar nuestro severo déficit presupuestario, que ha llegado a triplicar el que señalan los Pactos de estabilidad Presupuestaria de la UEM, y que aún hoy los duplica, también nuestro importante déficit tecnológico en bienes de alta y media tecnología que han llevado a una balanza comercial para este tipo de bienes deficitaria, impropia de un país como el nuestro. Nuestra dependencia energética, que se sitúa muy por encima de países próximos con los que competimos y que requiere de una regulación distinta, más abierta y flexible. También en el mercado laboral, donde sin duda se debe ser más prudente, es necesario menos complejidad y más flexibilidad.

Nuestra economía está todavía hoy, lejana a esa llamada "del conocimiento". El excelente informe ABACO del IVIE señala como en las economía más importantes y competitivas, el peso de las llamadas actividades basadas en el conocimiento están por encima del $75 \%$ de su PIB, mientras que en nuestro caso solo alcanzan el 55\%, lo que determina una mayor dificultad para un empleo estable (IVIE 2013).

Y como consecuencia de todo ello, la principal derivada que ha tenido y tiene esta crisis es la destrucción de empleo y la incapacidad para su recuperación, llegando al más triste de los datos, más de un $26 \%$ de paro, más que doblando la media Europea, y más de un 50\% de nuestros jóvenes menores de 25 años sin trabajo. Si bien, al menos en el caso de los universitarios, esta cifra se reduce prácticamente a la mitad.

De nuevo, insistir entonces en el interés y oportunidad del emprendimiento, pero no solo de cualquier emprendimiento. Nuestro Doctor honoris causa el filósofo Jose Antonio Marina con motivo de la presentación de su libro "la creatividad económica" en la que se apuesta por ella como elemento central para nuestra salida de la crisis decía: el emprender REVESCO $\mathrm{N}^{\mathrm{o}} 113$ - MONOGRÁFICO: Crisis económica actual y sus posibles repercusiones en la economía social - ISSN: 1885-8031 - www.ucm.es/info/revesco 
como trabajador autónomo es una solución de emergencia, el sálvese quien pueda" (Marina, Satrustegui 2013). No debemos basar la prosperidad solo en entidades como trabajadores autónomos por que no se va a desarrollar la técnica de alto nivel que es imprescindible. Necesitamos empresas que sean capaces de innovar.

Marina, sostiene también una visión del emprendimiento amplia no solo limitada al que busca empleo o al empresario y reivindica la necesidad de elevar el conocimiento económico de la sociedad.

Aquí, es bueno recordar que emprendimiento e innovación, siempre deben de ir acompañados de un buen plan de negocio y la adecuada gestión económica. De lo contario puede suceder como a la empresa económicamente señera de nuestro sector pesquero, PESCANOVA, que aun siendo una de las más referenciadas por su capacidad innovadora, atraviesa una delicadísima situación económica hoy, como todos conocen, y donde el informe forense de la consultora KPMG, habla de discrepancias entre la realidad y la contabilidad referida a la deuda financiera, sin duda fruto de una mala gestión económica y un mal plan de negocio.

En definitiva, aprovechar la oportunidad de que emerja un nuevo emprendimiento capaz de dotarnos de un nuevo tejido productivo, distinto, más creativo, más intensivo en conocimiento (científico, tecnológico y económico) y socialmente más comprometido.

Es en este punto, desde una valoración inicial positiva de la nueva ley de emprendedores, donde se debe señalar que si bien se contienen importantes incentivos y ventajas fiscales para éstos, especialmente en el inicio de la actividad, apoyo al emprendimiento como experiencia en la universidad, además de facilitar los trámites y reducir las responsabilidades personales del emprendedor ante posibles casos de quiebra, son disposiciones, aun comparativamente con otros países, insuficientes, y en nuestra opinión carentes de orientación y fomento de medidas más explicitas en el sentido de lo apuntado.

El pasado 5 de septiembre el periódico "el economista" hacía referencia a un informe de la consultora Bloomberg en el que señala a España como el País de Europa con peor pronóstico para los próximos cinco años, en referencia a la salida de la crisis por la lentitud en su proceso de recuperación.

REVESCO No 113 - MONOGRÁFICO: Crisis económica actual y sus posibles repercusiones en la economía social - ISSN: 1885-8031 - www.ucm.es/info/revesco 
Y el que estos malos augurios no se cumplan dependerá de que contemos con una política económica de crecimiento y empleo basada en el cambio de modelo que todos sostienen pero que no se acompaña de suficientes medidas, ni de la suficiente convicción en su lectura presupuestaria.

Una política hasta ahora centrada fundamentalmente en el ajuste presupuestario para la necesaria corrección del déficit, vía ingresos por el crecimiento de la carga tributaria (IRPF, IVA, IBI, especiales, etc..) y una drástica reducción del gasto público sin apenas distinción. Siendo que como señalan algunos expertos, lo que hemos de mejorar es sobre todo la eficacia recaudatoria más que la elevación de las cargas. Hoy la llamada economía sumergida, la no declarada, se estima, según el informe VISA (2013) de la consultora AT Kearney dirigido por el profesor Friedrich Schneider, que es el 18,6\%, que aun siendo verdad que ha disminuido y que se aproxima a la media de Europa (18,5\%), es bastante mayor que las de las economías más competitivas que están muy por debajo (ejm. Alemania 13\%, Francia y Reino Unido 10\%) (Figura 3), de hecho nuestra pérdida recaudatoria se cifra en unos 72.000 millones de $€$ como recogía el informe sobre la economía sumergida en España de la Fundación de Estudios Financieros (Jiménez, Martinez-Pardo 2013). También el informe VISA indica que sectores como la construcción, el comercio mayorista y retail, y el hostelero son los que mayores cifras dan de economía oculta (Schneider, Kepler, 2013).

REVESCO No 113 - MONOGRÁFICO: Crisis económica actual y sus posibles repercusiones en la economía social - ISSN: 1885-8031 - www.ucm.es/info/revesco 


\section{Figura 3}

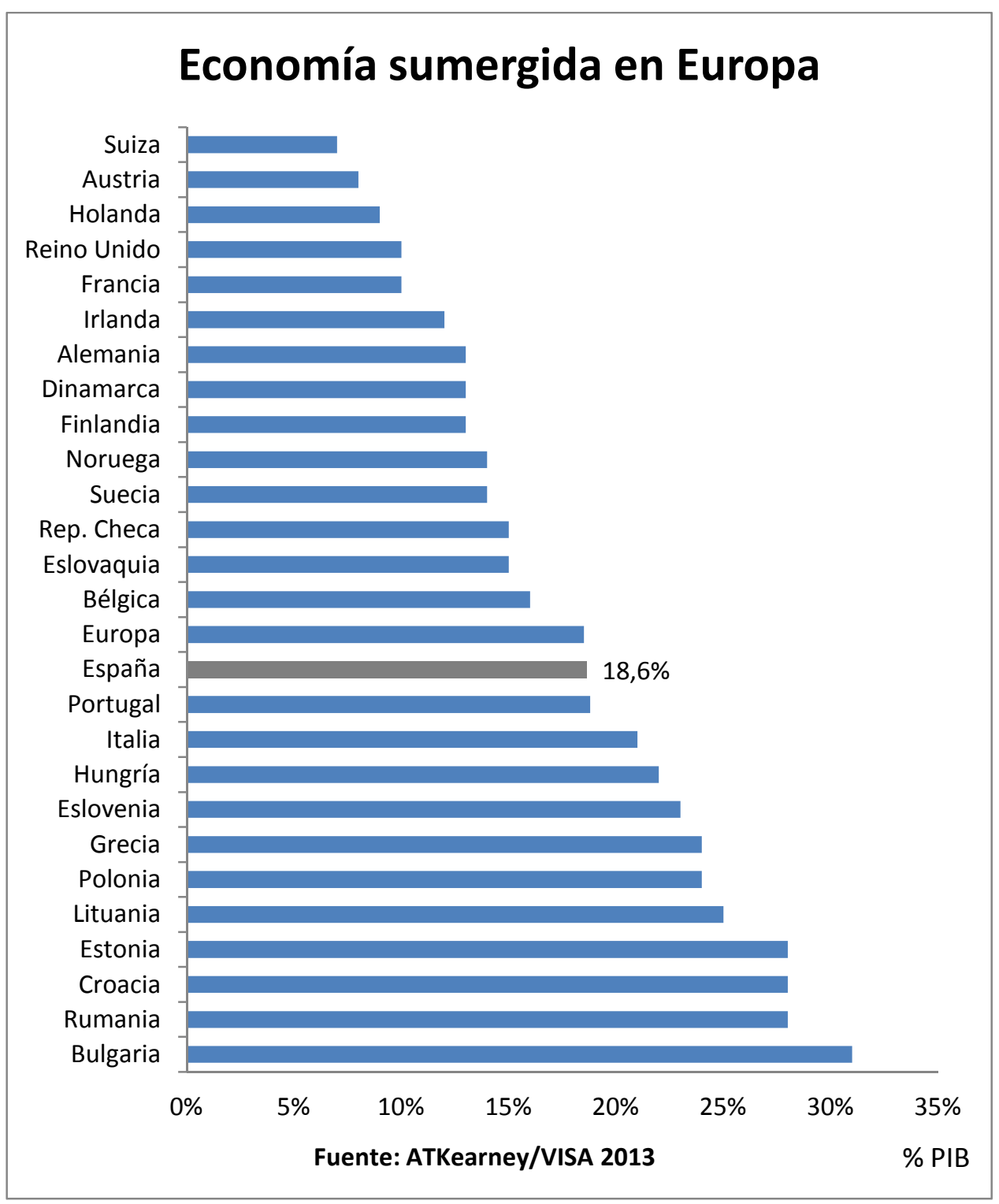

Una idea de la magnitud del problema en España, es que esta cifra supone en el 2009 el 74\% de nuestro déficit anual y que según la Fundación de estudios Financieros solo el que nos situáramos en un nivel de economía sumergida próxima a los países de nuestra entorno próximo contaríamos con una recaudación adicional de entre 18.000 y 20.000 millones de $€$ lo que equivaldría a una rebaja en nuestro déficit público nada menos que de 1,8 a $2 \%$ del PIB (Jiménez, Martinez-Pardo 2013).

De ahí la importancia de medidas para erradicar o al menos disminuir este problema, medidas que los expertos hablan, tanto de orden preventivo como sancionador con una regulación fiscal y laboral más clara y sencilla y especialmente con la mejora de nuestra

REVESCO No 113 - MONOGRÁFICO: Crisis económica actual y sus posibles repercusiones en la economía social - ISSN: 1885-8031 - www.ucm.es/info/revesco 
conciencia y moral tributaria, que bueno es señalar que afortunadamente según el citado informe está mejorando por parte de nuestra ciudadanía.

Otra de las medidas que necesitan de revisión y cambios es la reducción del gasto público, que no debe disminuir en el caso de capítulos tan estratégicos como la Educación superior y la $\mathrm{I}+\mathrm{D}$, como han hecho Francia y Alemania entre otros. Aquí al contrario se han producido importantes recortes. Solo según la CRUE en las Universidades Públicas Españolas su subvención ordinaria de 2009 a 2013 ha disminuido en 17\% en España (CRUE 2013), y en nuestra Comunidad Valenciana en un 19\%, llegando incluso la I+D a disminuir en los Presupuestos del Estado de 2009 al 2012 en un 26\%, que además ha ido acompañada de importantes disminuciones por parte del sector privado, así el último informe COTEC de 2013 llama la atención sobre que el número de empresas innovadoras de 2008 a 2011 se redujo un $43 \%$ y el de empresas con actividades en I+D en un 35\% (COTEC 2013).

Faltan medidas que impulsen también el ahorro energético y que disminuyan nuestra fuerte dependencia en cuanto a la partida de coste energético en nuestras empresas

Medidas que impulsen de forma efectiva nuestra transferencia de conocimiento, que desde luego no se corresponde con el nivel de nuestra producción científica donde como destacan distintos informes (Perez et al. 2012), representamos en torno a un $3 \%$ de la producción mundial, valor muy superior al peso de nuestras exportaciones de bienes y servicios, y cuando en patentes ni siquiera el $1 \%$.

Son tiempos de crisis, pero como Albert Einstein decía sin crisis no hay desafíos. Es el momento, la oportunidad de impulsar todas estas medidas para favorecer ese necesario emprendimiento innovador y generador de riqueza, pero los hechos y no las palabras parece que por el momento no acompañan.

\section{IMPORTANCIA DE LA UNIVERSIDAD EN EL EMPRENDIMIENTO}

Podemos decir, que por el solo hecho de que hoy más de un $40 \%$ del emprendimiento sea desarrollado por personas con educación superior y posgrados, justifica sobradamente que este tema sea de una especial atención en la política universitaria y en la actividad que las universidades desarrollan, y explica que en la nueva Ley de emprendedores se haya atendido a la conveniencia de promover las experiencias de emprendimiento en el marco universitario.

REVESCO No 113 - MONOGRÁFICO: Crisis económica actual y sus posibles repercusiones en la economía social - ISSN: 1885-8031 - www.ucm.es/info/revesco 
Pero ya hemos indicado que coincidiendo con la opinión de muchos expertos, el emprendimiento debe ser un instrumento para el desarrollo de la innovación, para que esta se produzca con el ejercicio de la actividad empresarial y este además orientada hacia el cambio de modelo productivo que basado en el conocimiento sea mucho más competitivo.

Desde luego siendo así, parece claro que el lugar más apropiado para generar un ecosistema más favorable a que este tipo de emprendimiento emerja, es la universidad, donde se producen 2/3 de nuestra producción científica en España, y en particular en el sistema público que concentra un $97 \%$ de esa producción y donde se forman más del $88 \%$ de nuestros titulados universitarios (CRUE 2010).

Es por ello que la Universidad debe entender la importancia del emprendimiento, que debe llevarlo a sus aulas y a su actividad universitaria en todos sus niveles de enseñanza y en especial debe impulsar y fortalecer así su tercera misión, que como saben es valorizar el conocimiento mediante una transferencia cada vez más activa con mayor compromiso con la innovación y el emprendimiento, con una permanente y estrecha colaboración con los distintos agentes económicos y sociales, empresas, Administraciones e instituciones.

El III Encuentro de Rectores UNIVERSIA RIO 2014 que se celebrará en esta ciudad brasileña en julio bajo el lema "La Universidad del siglo XXI" ha introducido como uno de sus debates esta cuestión, con la pregunta, ¿formamos en actitudes y capacidades creativas, innovadoras y emprendedoras?. Debate que modera el profesor David Noel Ramírez del TEC de Monterrey y que en sus conclusiones previas (Ramírez 2013), señala que independientemente del debate existente sobre la factibilidad para algunos de enseñar a emprender, no solo por cuestionar los métodos sino más aun al considerar el hecho de que se pueda desarrollar el espíritu emprendedor por ser algo muy inherente a la naturaleza de cada individuo, está la consideración de que los principales actores que determinarán el destino de la formación en emprendimiento, innovación y creatividad son las instituciones educativas a través de su profesorado y estudiantes.

Es nuestro deber impulsar el emprendimiento, desde un espíritu humano que busque la mejora continua de nuestra sociedad como sostiene David Bornstein, en su magnífica obra, "Cómo cambiar el mundo" que se editó por primera vez en ingles por la Oxford University Press en 2004 y que ha sido desde entonces traducida y editada en otras lenguas, y donde nos habla de los emprendedores sociales y el poder de las nuevas ideas (Bornstein 2009).

REVESCO $\mathrm{N}^{\circ} 113$ - MONOGRÁFICO: Crisis económica actual y sus posibles repercusiones en la economía social - ISSN: 1885-8031 - www.ucm.es/info/revesco 
También el informe BBVA/IVIE sobre "universidad, universitarios y productividad en España” destaca entre sus propuestas la promoción de la cultura emprendedora en la universidad (Perez et al 2012), lo mismo que los expertos que participan en la elaboración del GEM (Gomez et al 2009)

Hemos pasado de una enseñanza informativa (saber) a la basada en el saber hacer (adquisición de destrezas y habilidades) y ahora necesitamos desarrollar la capacidad de actuar de nuestro profesorado y alumnado, la llamada inteligencia ejecutiva y es verdad, que como muchos opinan ello exigirá cambios radicales en nuestro método educativo, y que además esto pueda contribuir también, desde un mayor compromiso social de la universidad, a aquello a lo que se refería James Grant director de UNICEFF desde 1980 a 1995, uno de los mayores emprendedores e innovadores sociales del pasado siglo, cuando decía, "la moralidad debe avanzar de la mano de la capacidad". Él que estudio en Harvard, siempre destacó por su compromiso social y su carácter emprendedor y fue quien concibió campañas mundiales con ideas imaginativas para poner a disposición de los niños de todo el mundo soluciones sanitarias sencillas a bajo coste, entre ellas las campañas de vacunación en muchas zonas en vías de desarrollo, que han salvado millones de vidas y a muchos niños de padecer daños irreversibles. Años más tarde la Fundación Bill\& Melinda Gates promovida por otro gran emprendedor del siglo XXI, con una ayuda de 750 millones de dólares apoya la extensión de esta actuación.

El experto en innovación, Xavier Marcet, con ocasión del seminario Universia, en el TEC de Monterrey, sobre ideas y oportunidades para el desarrollo de modelos de innovación vinculados a la universidad, señalaba que la universidad es un agente importante de los ecosistemas de emprendimiento e innovación, y puede ejercer de hub de encuentro entre inversores y emprendedores que pivotan en la universidad para crear empresas de alto valor. Puede ser no solamente una magnífica plataforma de emprendedores recién egresados sino también de emprendedores mayores y de dinámica de emprendimiento corporativo de empresas consolidadas (Marcet 2013).

Siendo, no obstante cierto, que queda mucho por hacer en este terreno en todas nuestras universidades, no sería justo dejar de reconocer que algo está ya cambiando. Hoy el emprendimiento y la innovación forman parte del discurso universitario, y se entiende como parte de la misión en muchas universidades, de su contribución al cambio de modelo

REVESCO No 113 - MONOGRÁFICO: Crisis económica actual y sus posibles repercusiones en la economía social - ISSN: 1885-8031 - www.ucm.es/info/revesco 
económico, a la generación de riqueza y progreso social, y especialmente por su relación con la mejora del empleo de nuestros egresados, buscando que esté sea lo más rápido, en mejores condiciones, más estable y adecuado.

De hecho, hoy felizmente podemos observar el importante esfuerzo realizado al respecto por nuestras universidades, solas o contando con la colaboración de algunas instituciones, si bien con un escaso apoyo hasta la fecha desde las administraciones.

Una buena muestra, son las experiencias recogidas en el libro que Redemprendia editó el pasado año, con el título,"100 buenas prácticas de emprendimiento universitario "donde se relacionan un centenar de casos de iniciativas y proyectos impulsadas por universidades iberoamericanas, un buen número de ellas españolas (Redemprendia 2013), como también los diez casos de éxitos que se analizan en la obra, "Emprender con éxito desde la universidad" (Romero et. al. 2013).

En este punto, nos gustaría destacar dos buenas iniciativas que están teniendo un excelente desarrollo.

Redemprendia, es una red de universidades creada en 2006, que promueve la innovación y el emprendimiento responsable desde el compromiso con el crecimiento económico, el respeto medioambiental y la mejora de la calidad de vida, en línea con sus universidades, entre las que se encuentran una veintena de universidades iberoamericanas entre las que están la mayor parte de las que más actividad de transferencia desarrollan. Dos de nuestras universidades públicas (Universitat de València y la Universitat Politècnica de València) ya forman parte activa de esta importante Red, que cuenta con el apoyo del banco Santander, institución financiera que a través de UNIVERSIA, la mayor red de universidades iberoamericanas del mundo y con más un millar de instituciones asociadas, muestra día a día su apuesta por el mundo universitario y el papel clave que desempeña para el progreso social y económico, y en particular en estos últimos años también por el emprendimiento universitario.

El Centro Internacional Santander Emprendimiento (CISE), es de más reciente creación, octubre de 2012, se trata de nuevo de una iniciativa impulsada por el Banco Santander, junto al Gobierno de Cantabria y la Universidad de Cantabria, que pretende constituirse en un centro de referencia del sistema universitario internacional en la

REVESCO $\mathrm{N}^{\circ} 113$ - MONOGRÁFICO: Crisis económica actual y sus posibles repercusiones en la economía social - ISSN: 1885-8031 - www.ucm.es/info/revesco 
investigación, transferencia y formación en emprendimiento, desarrollando proyectos docentes y actividades universitarias que estimulan la cultura emprendedora y la innovación, algunas de las cuales probablemente cuenten con un marco más adecuado con la entrada en vigor y desarrollo de la nueva Ley de Emprendedores.

El CISE cuenta con la valiosa colaboración entre otras instituciones, del Babson College, escuela de negocios de Massachusetts (Boston), que es un referente internacional en formación en emprendimiento. De hecho su programa master en administración de negocios ocupa el primer puesto en emprendimiento 19 años consecutivos por la US News\&World Report.

Una de las iniciativas del CISE, que más interés ha despertado es el programa e2, estudiante por emprendedor, un programa para el desarrollo de habilidades y el fomento de la cultura emprendedora, que sitúa a los participantes en una situación de emprendedor real.

Las universidades públicas valencianas a través de su CEI Hábitat 5U, constituirán en breve la primera antena/nodo regional del CISE, lo que permitirá desarrollar estas interesantes iniciativas en nuestro territorio y permitirá a los estudiantes de nuestras universidades y emprendedores participar activamente en programas como el e2, lo que sin duda es una buena forma de contribuir mediante el emprendimiento e innovación a la mejora de nuestro hábitat y territorio.

Se espera contar para ello en unos meses con la presencia del actual director del CISE y buen amigo de las universidades valencianas, el Profesor Federico Gutiérrez Solana, para el inicio del proceso mediante la firma de un acuerdo marco con las cinco universidades y su CEI Hábitat 5U. Iniciativa a la que deseamos se sumen todas las instituciones y empresas que en su día mostraron su apoyo explícito a este CEI., y de forma muy especial al Banco Santander. División Universidades quien como hemos visto no ha querido estar ausente en ninguna de las iniciativas emprendidas hasta la fecha con este fin.

Para terminar este punto, y dejando claro que todas nuestras universidades públicas vienen en estos últimos años desarrollando distintas iniciativas que pretenden impulsar el emprendimiento y la innovación, con algunas de las cuales he tenido ocasión de colaborar como con la cátedra INCREA de la UJI que dirige el profesor Francisco Michavila, permítame que me refiera a nuestra Universidad.

REVESCO No 113 - MONOGRÁFICO: Crisis económica actual y sus posibles repercusiones en la economía social - ISSN: 1885-8031 - www.ucm.es/info/revesco 
La Universitat Politècnica de València, es una universidad emprendedora e innovadora, la primera en este capítulo entre la españolas como reconoce el recientemente publicado U-ranking de la Fundación BBVA/IVIE (Fundación BBVA/IVIE 2013, ver (Tabla 1) u-ranking ISSUE- P de innovación y desarrollo tecnológico) y como muestra su trayectoria y el hecho de su liderazgo en transferencia, patentes y creación de spin-off. Viene apostando desde hace años por promover el emprendimiento innovador y de base tecnológica, apoyando y asesorando a nuestros emprendedores, a tal fin se creó en 1992 el Instituto IDEAS cuyo balance no puede ser sino satisfactorio. Más de 500 empresas creadas, 40/50 anuales en los últimos años, muchas de ellas por egresados e investigadores, y que han permitido también desde 2006 que la propia institución participe en algunas de las spin-off-UPV. Una labor complementaria a la del Instituto es la que vienen desarrollando la Cátedra de cultura directiva y empresarial promovida por nuestro Consejo Social y la Cátedra Bancaja Jóvenes Emprendedores.

Tabla 1: TOP 10 Universidades Españolas

\begin{tabular}{|c|c|c|c|}
\hline & Docencia & Investigación & Innovación \\
\hline 1 & Pompeu Fabra & Pompeu Fabra & $\begin{array}{l}\text { Politécnica } \\
\text { Valencia }\end{array}$ \\
\hline 2 & $\begin{array}{l}\text { Politécnica } \\
\text { Cataluña }\end{array}$ & Islas Baleares & $\begin{array}{l}\text { Politécnica } \\
\text { Cataluña }\end{array}$ \\
\hline 3 & Valencia & Barcelona & $\begin{array}{l}\text { Politécnica } \\
\text { Madrid }\end{array}$ \\
\hline 4 & $\begin{array}{l}\text { Autónoma } \\
\text { Madrid }\end{array}$ & $\begin{array}{l}\text { Autónoma } \\
\text { Madrid }\end{array}$ & $\begin{array}{l}\text { Pública } \\
\text { Navarra }\end{array}$ \\
\hline 5 & $\begin{array}{l}\text { Politécnica } \\
\text { Valencia }\end{array}$ & Valencia & $\begin{array}{l}\text { Autónoma } \\
\text { Madrid }\end{array}$ \\
\hline 6 & $\begin{array}{l}\text { Autónoma } \\
\text { Barcelona }\end{array}$ & $\begin{array}{l}\text { Rovira i } \\
\text { Virgili }\end{array}$ & $\begin{array}{l}\text { Autónoma } \\
\text { Barcelona }\end{array}$ \\
\hline 7 & $\begin{array}{l}\text { Complutense } \\
\text { Madrid }\end{array}$ & $\begin{array}{l}\text { Politécnica } \\
\text { Cataluña }\end{array}$ & $\begin{array}{l}\text { Santiago de } \\
\text { Compostela }\end{array}$ \\
\hline 8 & Islas Baleares & Lleida & Cantabria \\
\hline 9 & Salamanca & $\begin{array}{l}\text { Politécnica } \\
\text { Valencia }\end{array}$ & Alicante \\
\hline 10 & Lleida & Carlos III & Pompeu Fabra \\
\hline
\end{tabular}

Fuente: U. ranking 2013 Fundación BBVA/IVIE

Es cierto, aun con a ello, que también en nuestra universidad queda mucho camino por recorrer y que no podemos quedarnos satisfechos con lo mucho avanzado. Por ello expresar nuestra satisfacción porque el actual programa de gobierno del equipo rectoral haya

REVESCO $\mathrm{N}^{\circ} 113$ - MONOGRÁFICO: Crisis económica actual y sus posibles repercusiones en la economía social - ISSN: 1885-8031 - www.ucm.es/info/revesco 
introducido como un elemento central de su política universitaria la formación y el estímulo para el emprendimiento en todos los niveles formativos y en todas las estructuras, las creación de aulas a tal fin en los centros (Escuelas y Facultades), el ofrecimiento de formación y apoyo en emprendimiento también en posgrados y doctorados, así como la potenciación de IDEAS y la presencia de esa acción coordinada con las cinco universidades públicas desde la CPI en el marco del acuerdo CISE-Hábitat 5U, son en nuestra opinión iniciativas muy acertadas.

\section{HACIA UN EMPRENDIMIENTO INTELIGENTE Y CON VALORES}

De todo lo expuesto se desprende la importancia que tiene el emprendimiento para nuestra recuperación y progreso económico, pero de nuevo insistir en que siempre, un emprendimiento bien orientado que contribuya al cambio de modelo económico, a la llamada sociedad del conocimiento, un emprendimiento innovador, que como señala Federico Gutiérrez-Solana debe estar sustentado por el conocimiento como contenido, la creatividad y el talento como capacidad y el emprendimiento la actitud.

En relación con ésto, recordar una vez más al admirado nobel de economía y profesor en la Universidad de Columbia, Joseph Stiglitz, al que tuve ocasión de escuchar en Sevilla en el 2008, que señalaba que la actual gran crisis económica global, había puesto de manifiesto lo que él llamaba el fin del fundamentalismo de mercado, y que más importante que el origen de esta crisis tuviera lugar de la mano de algunos sectores (inmobiliario y financiero) era identificar que se trataba de una crisis de valores, que ha permitido como decíamos el imperio de la codicia sobre la razón y el bien común, por lo que reclamaba una mayor ordenación y supervisión de los mercados, sin por supuesto renunciar a la economía de mercado (Stiglitz 2010).

Necesitamos está claro, una economía diferente, basada en el conocimiento, que haga un uso inteligente del mismo, que incorpore creatividad, tecnología, que añada valor siendo realmente innovadora, pero con valores, como la cooperación, la sostenibilidad, el respeto medio ambiental, y el permanente compromiso ético, y siempre dentro de la economía declarada.

Una economía además plural, en la que coexistan lo público y lo privado tanto en las formas propias de la sociedad de capital tradicional como las de economía social y trabajo

REVESCO $\mathrm{N}^{\circ} 113$ - MONOGRÁFICO: Crisis económica actual y sus posibles repercusiones en la economía social - ISSN: 1885-8031 - www.ucm.es/info/revesco 
autónomo, la conocida también como la economía de rostro humano, donde prevalece el interés de las personas frente al capital.

Es en este sentido, superado el debate sobre la factibilidad o no de enseñar a emprender en la universidad, y reconociendo ello como una obligación más, que forma parte de nuestro compromiso social, que podemos afirmar que es la universidad, y en particular en nuestro país la pública donde se puede favorecer un ecosistema de emprendimiento orientado a la mejor respuesta a los importantes retos que tiene hoy la sociedad, entre ellos sin duda la recuperación de la economía y con ella del empleo, pero a la vez con visión de futuro y sin olvidar su papel en la promoción del emprendimiento social, aquel que David Bornstein reivindica por lo que representa para el cambio y mejora social y que puede ayudar a construir un mundo cada vez mejor..

Como conclusión, un emprendimiento inteligente y con valores. La Universidad debe hoy formar y generar conocimiento, transferirlo, pero hoy más que nunca ponerlo en valor mediante la innovación y el emprendimiento.

\section{BIBLIOGRAFÍA}

BARRO S. (2013) +Universidad-especulación. Ed. Netbiblo . La Coruña pp 171

Bornstein D. (2009) Como cambiar el mundo. Ed. Debate. L'Hospitalet. pp447

COTEC (2013) Informe Cotec 2013. Tecnología e innovación en España (www.cotec.es)

CRUE (2010) La universidad española en cifras. (www.crue.org)

DRUCKER P. (1997) Capitalismo, socialismo, democracia. Ed. Aguilar. Madrid pp512

FUNACIÓN BBVA/IVIE (2013) u-ranking de universidades españolas. (www.u-ranking.es)

GEM (2012) Informe GEM España 2012. Global entrepreneuship Association. (www.gemspain.com)

GOMEZ J. M., MARTINEZ J. M., MIRA J., (2009) caracterización del emprendedor de la C.V.: elementos sociodemográficos, capital humano y social, y factores situacionales. Ed. UMH. Cátedra Bancaja jóvenes emprendedores. pp139

GUTIERREZ-SOLANA F. (2013) Conocimiento, creatividad, Emprendimiento e Innovación. Rev. Tribuna complutense. Opinión pag 4

IVIE (2013) Observatorio de las actividades basadas en el conocimiento. Informe ABACO 2013 (www.observatorioabaco.es)

REVESCO No 113 - MONOGRÁFICO: Crisis económica actual y sus posibles repercusiones en la economía social - ISSN: 1885-8031 - www.ucm.es/info/revesco 
JIMENEZ A., MARTINEZ-PARDO R. (2013) La economía sumergida en España. Documentos de trabajo n ${ }^{\circ}$ 4. Ed. Fundación de Estudios Financieros. pp 94

MARINA J.A., SATRUSTEGUI S. (2013) La creatividad económica. Ed. ARIEL. Barcelona pp196

PEREZ F. et al (2012) Universidad, universitarios, y productividad en España. Fundación BBVA/ IVIE

RAMIREZ D. N: (2013) III Encuentro de Rectores UNIVERSIA-RIO. Debates previos) (www.universia.es)

MARCET X. (2013) Universidad, innovación y emprendimiento (www.Xaviermarcet.com/2013/06/universidad-innovación-y-emprendimiento)

REDEMPRENDIA (2012) 100 buenas prácticas de emprendimiento universitario. 486

ROMERO J. M. et al (2013) Emprender con éxito desde la universidad. Colección estudios redemprendia. Ed. Netbiblo. La Coruña pp 117

SALABURU P. (2006) El modelo de universidad americana, i es un referente para Europa. EN TOLEDO F., ALCON E., MICHAVILA F. Universidad y economía en Europa Ed. Tecnos. Madrid pp 100-134

SCHNEIDER F., KEPLER J. (2013) The Shadows Economy in Europe. Informe ATKearney /VISA

SCHUMPETER J. (1968) Capitalismo, socialismo, democracia Ed. Aguilar Madrid pp512

SOLOMONT A. D. (2013) Porque florecen los emprendedores en Estados Unidos. Rev Executive Excelence $n^{\circ} 78$ pp 1-4

STIGLITZ J.E: (2010) Caída libre: el libre mercado y el fundamentalismo de la economía de mercado. Ed Taurus. Madrid pp 423

REVESCO $\mathrm{N}^{\mathrm{o}} 113$ - MONOGRÁFICO: Crisis económica actual y sus posibles repercusiones en la economía social - ISSN: 1885-8031 - www.ucm.es/info/revesco 